\title{
Organization of distance learning on the base of information and digital technologies
}

\author{
Tatyana Shkil ${ }^{1, *}$, and Tatyana Belikova ${ }^{1}$ \\ ${ }^{1}$ Don State Technical University, Gagarin square, 1, Russia Rostov-on-Don, 344000, Russia
}

\begin{abstract}
The need for digital transformation of education in Russian Federation presupposes the development of new teaching methods, including online methods, information and digital interactive technologies and communication tools. All universities in Russia switched to distance learning due to the quarantine in the spring of 2020 and that was a kind of testing ground for testing effectiveness of these methods. The purpose of the article is to summarize the experience of distance learning of all types of classes in the discipline "Physics". Analysis of this experience and the results of approbation of various teaching methods and interactive technologies allows to identify the optimal methods for conducting classes of various types online and the technical solutions for their implementation. This article provides systemic information on the arranging the distance learning in the discipline "Physics" at the Don State Technical University and gives specific recommendations on the methodology for all types of classes and on the organization of control over the assimilation of the material by students. The effectiveness of these methods is confirmed by the results of the exam session of full-time and part-time students.
\end{abstract}

\section{Introduction}

The transfer of educational organizations in Russia to distance learning, due to the quarantine in the spring of 2020, clearly demonstrated the need for digital transformation in the education.

In connection with the quarantine introduced because of the coronavirus infection, starting from March 16th, 2020 the country's universities have gradually switched to distance learning. With this form of education, it becomes necessary to provide all students with learning and methodological materials to conduct remotely all types of classes; and these materials should be produced in digital form.

For distance learning of students of Don State Technical University (DSTU), it was possible to use the DSTU SKIF platform, online courses, any available services and platforms for video conferencing or webinars (Skype, Zoom, etc.). For communications any convenient messenger could be used. Another possible option is to use the massive open online courses (MOOCs) from the list recommended by the Ministry of Science and Higher Education, if these MOOCs fully correspond to the disciplines studied.

\footnotetext{
*Corresponding author: tanashkil@mail.ru
} 
According to the results of Russian researchers [1, 2], in Russian universities, the demand for MOOCs is greater among professors than among students, especially the students with high academic performance. In addition, the number of works devoted to studying the MOOC market and the prospects for their implementation into the existing education system is relatively small. There is a discrepancy in assessing the results of similar test items for different MOOCs, often the scores are overestimated [3].

In the studies of a number of foreign authors it is noted that massive open online courses are an example of the introduction of innovative technologies not in the education domain, but in fact in the business domain, since the introduction of MOOCs gave the universities almost unlimited opportunity to increase the number of students only through the use of the digital environment [4]. Many MOOCs are aimed at those who are unable to pay for regular expensive education, do not have time for traditional education, as well as at those for whom online courses are an addition to the already received basic education [5]. Implementation of open educational resources in the educational environment makes it possible to significantly reduce the education cost [6]. The cost of online education based on MOOCs for bachelor degree course is about $75 \%$ less than the cost of traditional fulltime education [7]; so the MOOC technologies expand access to quality education for almost everyone [8]. The authors [9, 10] believe that at present time the entire system of higher education needs to be changed and the implementation of the changes, in particular, should take place on MOOC platforms, which can serve as a kind of laboratories for various research projects in the pedagogy domain. The psychometric aspects of online education are also considered, in particular, the relationship between the intelligence of students and the degree of their readiness for online learning [11]. The works of Russian authors, as a rule, analyze the problems of transition of the national education to the digital educational environment $[12,13]$.

As the analysis of the publications shows, the forced transfer of the full-time education to the distance learning have no analogues in the teaching practice of higher education; a detailed description of the methods and technologies for conducting all types of distance learning in various disciplines are not generally available; so the use of MOOCs, especially in the first year, is unlikely and ineffective. That is why switching to distance learning has demanded that professors develop new methods and forms of teaching and working with students. The skills of remote transmission of information and online teaching, information about large interactive educational portals and a "guide" to all open online resources were also needed.

\section{Methodology}

All educational organizations switched to the distance learning during the quarantine. This option has become a kind of effective "testing ground" for modern online teaching methods, information and digital interactive technologies and communication tools. Obviously, the experience of their use in the course of distance learning and the conclusions and recommendations made on its basis are needed to be systematically analyzed.

The purpose of the article is to summarize the experience of distance learning of all types of classes in the discipline "Physics". Analysis of this experience and the results of approbation of various teaching methods and interactive technologies allows to identify the optimal methods for conducting classes of various types online and the technical solutions for their implementation.

Distance learning involves conducting classes on a schedule, though not in classrooms, but on online platforms, so the professors must use new ways to deliver the learning materials and to test the knowledges. Seminars and lectures are replaced by conferences or webinars, knowledge control is carried out by testing, and by review the solved tasks in the 
students' workbooks (sent by e-mail or WhatsApp). The presentations are presented and discussed in Skype or Zoom. One of the main problems is the examination and passing tests. There are various options: oral survey; remote testing on a schedule; a combination of testing and oral communication. All the above methods create problems for a number of reasons: in a synchronous approach (scheduled exam), problems with Internet speed are possible; in case of online testing, it is impossible to ensure proper control over students.

To communicate remotely, the professors and students needed a personal computer with a standard text editor, a webcam and a microphone (or a mobile device) and a stable Internet connection.

The greatest difficulties arose for the professors of technical and natural sciences, in particular, physics, where the learning materials contain numerous formulas, complex

mathematical calculations, diagrams, graphs, drawings, and laboratory practice is expected. In this case, only online teaching is not enough, since checking tasks for practical and laboratory classes requires to adjust various methods to give feedback and to evaluate the results obtained. The distance learning makes necessary to issue various kinds of creative tasks to students and then review them, i. e. long-term work with students in close contact. In addition, for any university, transferring laboratory courses in natural sciences, where students conduct experiments, into online mode is not an easy problem.

Traditionally, physics classes are held in the form of lectures, which present theoretical material, and practical classes of two types: solving problems and performing laboratories; for each of the listed types of classes, there is a task for students for independent study. When switching to distance learning for students, the detailed program of classes was developed until the end of the semester in accordance to the schedule of classes. For each class hour, the topic and plan of the class, numbers of problems to be solved and laboratories performed, tasks for independent work, references to literature or other sources of information were given. The program was posted on the website of each students group and in the group chat in WhatsApp, i.e. brought to the attention of each student. Then it was implemented during the educational process.

Theoretical classes (lectures) aimed to equip students with a system of professionally important knowledge were conducted in the form of online conferences on the Zoom platform. Based on the electronic lecture notes in physics, which were provided to all students at the beginning of the semester, a presentation was prepared for each lecture. The presentation was used during the class hours. The theme and plan of the lecture, developments of formulas and mathematical records of physical laws, drawings, diagrams and graphs were presented on the slides of the presentation. All texts - an explanation of the used models, concepts, mechanisms of the phenomena under consideration, the formulation of laws, definitions, etc. was presented by the professor orally during the class hours. Since the students knew the topic of the lecture in advance and had to familiarize themselves with the studied material, at the end of the class a part of the time was reserved for answering questions if some moments of the lecture were not fully understood.

When studying the discipline "Physics", the greatest difficulties for students are to solve physical problems, since formal knowledge of physical laws is not enough for this. So, it is necessary to possess special methods and algorithms for solving problems of a certain type. In some cases, it is not possible to develop such algorithms and methods, and then the decisive factor is understanding the physical essence of phenomena and laws that allow to solve the problem, as well the ability to think analytically.

In-depth study of the discipline, as a rule, occurs during practical classes and preparation for them. In the class hours the students start to comprehend the physical laws, to understand their practical significance when solving general scientific and engineering problems. They form skills to choose the optimal solution path and to make mathematical recording, to evaluate the result obtained, i.e. in particular the creative abilities of students 
are developed.

With distance learning, practical classes in physics take a certain specificity associated with the need to use information technologies. Analyzing our own experience, we can definitely state that conducting classes on solving problems in a distant mode requires to change the methodology of their organization and to transform the methodological base into an on-line format.

For the successful implementation of the practical classes, it is necessary to familiarize the students with a detailed class plan and recommendations how to prepare for the class. The professor defines a range of theoretical questions, a list of concepts, definitions, laws and formulas with links to textbooks and methodological manuals posted in the University electronic learning information system. Students are provided with examples of solving problems on this topic with a detailed analysis of the methodology, which must be studied before the class, and with a list of a sufficient number of problems that can be solved by students in class based on the studied examples. Practical preparation recommendations are sent via WhatsApp or email. Students receive information in advance, a few days before the class, and at the preparatory stage they prepare for the class on their own. This stage of preparation of the lesson requires a lot of time from the professor to create high-quality methodological support.

To conduct classes on solving problems, in particular remotely, it is reasonable to use special problem books. These methodological manuals first present a brief theory and methodology for solving problems in specific section of physics. Then detailed solutions for all typical problems are given, and only then similar problems are proposed to solve independently. Such a presentation of the material allows most students to independently learn to solve problems, since the methodology for solving problems, which is different for different course sections, is actually an algorithm of the student's actions. This is especially important if a student has missed previous lectures or practical classes for any reason, cannot identify the basic formulas without assist, even does not understand how to start solving the problem, and does not have the necessary knowledge and skills in mathematics. Solving typical problems according to the proposed model allows students to overcome these difficulties and to acquire the skills of independent problem solving.

One of the possible scenarios for conducting a practical class remotely using such a problem book is as follows. At the beginning of the semester, all students are provided with a problem book (in electronic form). Then during the class, the professor orally briefly recalls the basic physical concepts, laws and formulas on the topic under study, referring to the material in the book or using a presentation. The slide contains a list of numbers of solved problems (examples of solutions), a list of typical problems for independent solution and, finally, numbers of non-standard problems or problems of increased complexity for the most powerful students. Then, the group examines in detail the methodology for solving problems in this specific section using the example of one of the typical problems. Further, the students independently analyze the example solutions of problems and try to solve similar problems, if necessary, consulting with the professor. At the same time, powerful students can go ahead of the group and after study the solution of typical problems they can move on to problems of increased complexity.

The practical class itself involves direct communication between the professor and students using on-line tools, for example, Zoom platform or Skype. During the class, when discussing a problem or answering students' questions, the professor may need to quickly demonstrate the progress of the solution on the computer display. However, the solution of a physical problem involves the presentation of diagrams, drawings, graphs and writing formulas, which is difficult to quickly implement "using keyboard". However, it is possible to demonstrate pre-prepared slides where a problem is solved or write the solution on a sheet of paper before a camera, or demonstrate the solution using a raster graphics editor 
Microsoft Paint. But drawing and writing using mouse is inconvenient, and the images cannot be drawn with very high quality. It is also possible and effective for the professor to use a tablet computer in combination with a stylus for handwritten information output on a practical class or at a lecture.

The volume of independent work of students significantly increases with distance learning, while the load on the professor for its organization and control over its implementation significantly increases. Experience has shown that an effective type of independent work of students in the study of the "Physics" discipline is now the implementation of computational and graphic works, involving the solution of multistage problems, performing calculations and plotting the dependences of physical quantities. The implementation of such works certainly contributes to the development of students' creative abilities, motivates them to participate in research work, prepares them to complete the assignments of term papers and theses. The students took photos of the completed works and sent them to the professor by e-mail in JPG format, which allows to put notes and comments on the image when assessing the work. To reduce the time spent by the professor for communication with the student, it is possible to use, for example, the Jing Project software for audio commenting.

The purpose of the laboratory works in physics is to consolidate the theoretical knowledge acquired at the lectures and to acquire practical skills in using measuring equipment. During the labs the students get acquainted closely with the studied physical phenomena, the most important methods of experimental research and determination of a set of physical quantities. They experimentally check the correctness of physical laws, acquire the skills to make an experiment and to perform statistical analysis of the results.

The laboratory classes of the Department of Physics of DSTU is a high-tech complex. On its basis it is possible to perform more than one hundred and twenty different labs in all studied sections of the discipline "Physics". The complex is equipped with modern multifunctional equipment from PHYWE, which involves performing a number of labs and processing the obtained experimental data using software. Each laboratory work is provided with a methodological manual and a form for recording experimental data and their subsequent statistical analysis in digital form. The methodological manual contains all the information necessary to perform the work: the purpose of the work, a brief theory, a detailed description of the experimental setup, including its individual elements and their functional purpose, the methodology and procedure for performing the experiment, formulas for calculations, necessary tables, a list of used literature and control questions. The form contains tables to write the experimental data and parameters of the setup, formulas for calculating physical quantities and their errors. There is a field to perform the graphic part of the work (to build various plots), to write the final result of calculations and to analyze the results of the work.

In the case of distance learning on-line [14], it is most difficult to organize laboratory classes in physics in such a way that all the set educational goals are achieved during the class. In the situation of distant learning the authors used various scenarios and methods of laboratory classes with the involvement of the unique material and technical base of the Department of Physics.

To conduct laboratory classes on-line, a video of a number of laboratory works was recorded. The recording was carried out in the film studio of the information service of the DSTU and based on the self-recording studio for the DSTU online courses. The video of one laboratory work lasts approximately 12-14 minutes and provides complete record of the professor's work on the laboratory setup. At first, the professor reminds the safety rules for performing laboratory works, then formulates the topic and purpose of the work, notes its relevance and practical significance, gives examples of the use of the studied phenomenon in science and engineering, reminds the main laws revealed in the course of the lab. Then 
the laboratory equipment is described in detail; the functional purpose of all elements of the laboratory setup, the principle of its operation, features and procedure for performing the work are explained; the methodological description of the lab and the corresponding input form with the necessary explanations are also demonstrated.

After that the professor performs an experiment and demonstrates its result before camera. When determining the time, the light indicators of the electronic stopwatch are shown, when determining the distances, the measuring scale with the corresponding experimental marks are demonstrated, etc.

Some laboratory works were performed using a personal computer with appropriate software for each work. In this case the results of the experiment were displayed in the form of graphs and tables of experimental data. Then the data were used by students for subsequent statistical analysis to obtain the final results of the work. Wacom Cintiq Pro graphic tablet was used during the recording to display this image on a large screen which was exposed to camera. So, the professor could comment the results of the experiment and give instructions for further work of students with the data obtained. The stylus allowed the professor to mark on the screen the characteristic points of the plots, to highlight individual parameters, etc., that is, to operate with the screen. The screenshot is an attachment to the video.

Laboratory classes in normal conditions are conducted in such a way that students receive individual tasks, i.e. either carried out different laboratory work on given topic, or the same work was performed on different setups or on the same setup under different conditions (i.e., some parameters of the setup were varied, for example, the distance from the diffraction grating to the screen). In order to ensure the tasks were individual in distance learning, ten different experiments were performed in advance on one setup under different conditions (for different values of a certain setup parameter). For example, when determining the acceleration of gravity the length of the mathematical pendulum changed, when determining the moments of inertia of bodies of regular geometric shape bodies of various masses and shapes were studied (ball, solid and hollow cylinders, disk and rod), when determining the kinematic characteristics of translational and rotational movements the body path changed, etc.

The results of the experiments were summarized in the table, which allows to give students individual tasks; the number of the task option is determined by the last digit of the student's number in the alphabetical list of the group. If the work was carried out using a laptop with the lab software, then the options for tasks were formed on the basis of graphs and tables of experimental data displayed on the laptop screen.

Thus, for one laboratory class, a kit was formed consisting of a video recording, a methodological description of the work, an input form for performing the lab, tables of options for tasks and experimental data, and a laptop screenshot if the work was performed using the software. Such a kit allows to clearly and easily present the necessary learning information and use the class time as efficiently as possible. These methodological kits for conducting laboratory classes are posted on the website of the Department of Physics of DSTU on YouTube. In addition, methodological instructions for performing the work, a form for laboratory work and experimental data can be found via the link.

Students must prepare for the class, i.e. read the methodological description of the work, watch the video, if possible, print the form of the work (or fill it out in electronic form), write in the form the experimental data of their option of the task. It is reasonable to conduct the class using the Skype or Zoom platforms, while using e-mail or WhatsApp in parallel to review the forms. Typical errors identified during the review can be immediately discussed with the entire group. Knowledge of theoretical material can be reviewed either by testing on the SKIF DSTU platform, or in the form of an individual oral survey using any convenient service. 
Along with the "video clips" of laboratory work, a virtual works were used for the handon practice: the licensed physics labs by "Physicon" company purchased by DSTU and virtual works created with the participation of the department professors. The Department of Physics has a large bank of virtual laboratory works in all sections of physics. In a virtual laboratory work the operation of physical instruments or real physical process is simulated. The advantages of virtual laboratory work include the ability to see the operation of equipment that are not available in a university laboratory; the safety of work, for example, with high voltages, high currents or radioactive substances; elimination of possibility to break expensive devices. Using computer physics labs and changing the parameters of the experiment, it is possible to make the work of each student absolutely individual. Gradually raising the experiment complexity, the professor can consistently improve the students' skills in modeling the experiment.

Virtual laboratory work can be considered as one of the ways to form the research competence of a future specialist. Properly constructed virtual experiments can become a good motivation for the students to develop creative thinking and they can help to form skills in organizing research work. This inevitably leads to the development of the ability to predict various processes, which is of great importance for the professional activity of an engineer.

Experience has shown that a reasonable combination of various methods of conducting laboratory classes allows students to maintain the same high interest in a physical experiment as in a real labs. The active interaction of professors and students in the on-line laboratory work can give good results in developing students' skills for planning an experiment and mathematical processing of its results. However, when studying natural sciences, it is impossible to completely refuse natural experiments and practical work with instruments.

Organization of control over the assimilation of the learning material by students is a particular difficulty in distance learning. Organization of written tests, defense of laboratory work and passing an exam (test) requires a careful development of the rules for interaction between a professor and a student. When carrying out control activities, the authors tested the three most common methods: oral survey; written test; remote testing. All these methods have their own advantages and disadvantages.

Oral survey can be carried out in real time via video communication, as if it were taking place in a university classroom. In this case, it is optimal to form small groups of 3-4 people and assign each of them a time to get online. It is necessary to think in advance about the rules of student behavior during the preparation and the survey itself (do not interrupt the video communication; readiness to show your computer screen at any time; the ability to use a synopsis or textbook, etc.) and sanctions from the professor if a violation of the rules is noticed. With this form of control, in addition to listening to the prepared answer, it is possible to ask the student unexpected questions that allow to assess his level of knowledge, understanding of the material, the ability to analyze and to link the material of various topics of the studied discipline. However, an on-line oral survey, as experience has shown, is very time-consuming, and often does not fit into the hours of the planned classes.

In the written form of the knowledge control the student performs the work in writing and sends it to the professor for review. In order to exclude the element of "cheating", it is necessary, as experience has shown, to fulfill the following conditions: to send tasks immediately before the start of control and to strictly limit the time of execution and delivery of work. In addition, the control tasks should be exclusively individual and include elements of engineering work. Fulfillment of the latter condition required the creation of multivariant tasks that would allow to control not only the basic physics knowledge of students, but also the formation of their abilities and skills to apply this knowledge to solving problems of different complexity. Like the oral form of control, the written control 
works require a significant time spent to review them, describe and comment the errors and to justify the marks.

For testing, the Department of Physics on the SKIF DSTU platform created a quite large bank of tasks for all sections of the discipline. The service allows the professor to track the number of student attempts to pass the test, the time spent by each student, the final score and questions with the wrong answers. If there is a bank of tests, testing does not take much time to organize. If testing is carried out simultaneously for the entire student group (course) and the time to pass the test is limited, then the possibility to cheat when passing the test can be excluded.

To identify the student and to guarantee compliance with the rules for passing the exam, universities began to use "proctoring" technologies, namely observing students during a distance exam (from the verb "proctor"). The proctoring system can be implemented in two versions: the examinee is observed by a person through the monitor screen, or a special software program is used to control the "undesirable" behavior of students. Proctoring technology has been used for more than ten years in leading universities in the world and in Russia [15]. The use of proctoring services excludes any interest in the results of passing the exam, frees the professor from the need to monitor the behavior of several students simultaneously during the exam. In addition, it motivates students to prepare for the exam honestly. Switching of any university to full or partial distance education, in our opinion, will inevitably lead to the use of proctoring.

\section{Conclusions}

The effectiveness of the considered distance learning methods can be estimated based on the rating of students and the results of the exam session during such learning: all students who attended the online classes successfully passed the exam, and their marks in the most cases appear to be the same as in the winter exam session. Similar results were shown by correspondence students, whom classes were also held remotely. The methods used have shown their effectiveness, allowed to rationally use the classes time, and appeared to be comfortable for students.

The accumulated experience of distance teaching of physics indicates that it is recommended to read lectures using slides containing a minimum of text material; for practical exercises it is reasonable to use special problem books with examples of solving typical problems; and during laboratory exercises, professors should use a kit containing a video of the laboratory work and the necessary methodological support. To organize the control of the assimilation of the material by students, to use proctoring technologies is recommended.

Distance teaching is much more labor consuming compared to other forms of education, since an extremely detailed organization of classes is required: those points that are usually explained and discussed during the class directly in the classroom must be described in detail and clearly on the website of the discipline. The professors' workload increases significantly, since conducting classes on the Internet requires careful and long preparation: the development of slides and presentations, videos for laboratory classes, mailing materials, etc. In addition, a huge amount of time is required to review individual tasks for independent work in practical and laboratory classes.

It is obvious that the temporary switch of the entire national educational system to distance learning was a kind of test of the ability of educational institutions to use modern innovative technologies for organizing and implementing the educational process. At the same time, this motivates to implement such technologies and to explore their possibilities to be a base for the education system reform. Undoubtedly, the implementation of digital technologies will inevitably change the didactics (the science of forms, instruments and 
methods of teaching), but it will not replace the need to think, that is, to comprehend and analyze the information received, correlate information from various sources, critically evaluate it and exclude unreliable information. Distance learning and the corresponding innovative educational technologies are permanently embedded in the modern education system, but it should be remembered that success in distance education depends to a large extent on the student's performance: his or her motivation, commitment, perseverance and self-discipline.

\section{References}

1. Y. Roshchina, S. Roshchin, V. Rudakov, Educational Studies, 1, 174-199 (2018) DOI: 10.17323/1814-9545-2018-1-174-199

2. T. Semenova, K. Vilkova, I. Shcheglova, Educational Studies, 2, 173-197 (2018) DOI: 10.17323/1814-9545-2018-2-173-197

3. D. Kravchenko, Educational Studies, 4, 99-115 (2018) DOI: 10.17323/1814-95452018-4-99-115

4. S. Young, Educational Studies, 4, 21-43 (2018) DOI: 10.17323/1814-9545-2018-4-2143

5. L. DeBrock, Educational Studies, 4, 44-59 (2018) DOI: 10.17323/1814-9545-2018-444-59

6. D. Keyek-Franssen, Educational Studies, 4, 116-138 (2018) DOI: 10.17323/18149545-2018-4-116-138

7. S. Ludwig, Educational Studies, 4, 167-187 (2018) DOI: 10.17323/1814-9545-2018-4167-187

8. R. Stein, Educational Studies, 4, 188-198 (2018) DOI: 10.17323/1814-9545-2018-4188-198

9. Q. McAndrew, Educational Studies, 4, 60-80 (2018) DOI: 10.17323/1814-9545-20184-60-80

10. M. Janelli, Educational Studies, 4, 81-98 (2018) DOI: 10.17323/1814-9545-2018-481-98

11. A. M. Alenezi, The Education and science journal, 4(22), 89-109 (2020) doi.org/10.17853/1994-5639-2020-4-89-109

12. O. Usacheva, M. Chernyakov, Higher Education in Russia, 5(29), 53-62 (2020) doi.org/10.31992/0869-3617-2020-29-5-53-62

13. E. Brodovskaya, A. Dombrovskaya, T. Petrova, R. Pyrma, A. Azarov, Higher Education in Russia, 12(28), 9-22 (2019) doi.org/10.31992/0869-3617-2019-28-12-922

14. C. S. González-González, A. Infante-Moro, J. C. Infante-Moro, Sustainability,12(8), 3488 (2020) doi.org/10.3390/su12083488

15. A. M. Reagan, Journal of the Washington Academy of Sciences, 98(1), 31-46 (2012) https://www.jstor.org/stable/24536566 